\title{
Ocular manifestations in patients with systemic sclerosis
}

\author{
Małgorzata Kozikowska1, Wojciech Luboń², Eugeniusz J. Kucharz ${ }^{3}$, Ewa Mrukwa-Kominek ${ }^{2}$ \\ ${ }^{1}$ Department of Ophthalmology, Professor K. Gibinski University Clinical Center of the Medical University of Silesia, Katowice, Poland \\ 2Department of Ophthalmology, Faculty of Medical Sciences in Katowice, Medical University of Silesia, Katowice, Poland \\ ${ }^{3}$ Department of Internal Medicine, Rheumatology and Clinical Immunology, Medical University of Silesia, Katowice, Poland
}

\begin{abstract}
Systemic sclerosis (SSc) is a rare, chronic autoimmune disease with unknown etiology. Its prominent features are fibrosis, vasculopathy and impaired immune response. Disease can also affect eyes leading to various findings in ophthalmological examination.

The objective of this study was to determine the prevalence and type of ocular involvement in patients with SSc. A systematic literature review was conducted using electronic databases. A combination of following keywords was used: "systemic sclerosis" and ophthalmology-related search terms, including the keywords "eye", "ocular" and "ophthalmic".

In conclusion, eyelid and conjunctival abnormalities and dry eye disease are among the most common ocular manifestations of SSc. Their diversity is connected to complexity of the disease.
\end{abstract}

Key words: systemic sclerosis, dry eye disease, ocular manifestations, eyelid changes.

\section{Introduction}

Systemic sclerosis (SSc) is a rare, severe systemic disease of unknown etiology and little-known pathomechanism. The disease is characterized by vascular changes, autoimmune disorders and progressive fibrosis of the skin and internal organs [1, 2]. There is a limited form of the disease (ICSSC - limited cutaneous systemic sclerosis), in which the occurrence of skin lesions far exceeds the development of internal organ fibrosis and a generalized, diffuse form (dcSSc - diffuse cutaneous systemic sclerosis), in which internal organ involvement occurs shortly after the appearance of skin lesions. The location of skin lesions in both forms of the disease varies and they are characterized by the presence of other autoantibodies.

Rarely, SSc is characterized by the development of organ lesions without the appearance or late manifestation of skin lesions, i.e. systemic sclerosis sine scleroderma [3]. There are also SSc overlap syndromes with other systemic connective tissue diseases, most often with polymyositis (scleromyositis). Mention should also be made of a very early systemic sclerosis, which is a pre-systemic sclerosis condition characterized by
Raynaud's phenomenon, puffy fingers, disease-specific autoantibodies, and microvascular alterations at capillaroscopy [4]. It is estimated that in $65-80 \%$ of patients with very early systemic sclerosis the full clinical profile of the disease will be revealed within 5 years. It is unclear whether SSC is a homogeneous disease and is a nosological unit or is a syndrome with differentiated etiopathogenesis and only a common final clinical presentation.

The organ of vision is largely composed of connective tissue structures. This is a premise suggesting that ocular changes may occur in patients with SSc. In addition, the discussed disease substantially affects blood vessels.

Vascular damage primarily involves microcirculation, which is expressed by the early development of capillaroscopic visualized lesions, but may also involve larger vessels. Typical is Raynaud's phenomenon, which may also have organ counterparts. For example, myocardial ischemia is observed in patients which is not related to the development of atherosclerosis, but having the nature of transient coronary vasospasm. In the renal and other arteries (e.g. finger arteries) a hyperplasia of muscle layer that reduces or closes the lumen of the vessel is developed. In the case of renal arteries, this can lead

Address for correspondence:

Małgorzata Kozikowska, Department of Ophthalmology, Professor K. Gibinski University Clinical Center of the Medical University of Silesia, 35 Ceglana St., 40-514 Katowice, Poland, e-mail: kozikowskamalg@gmail.com

Submitted: 15.06.2020; Accepted: 24.11.2020 
to renal crisis, which was the main cause of death for SSc patients for many decades. To what extent this phenomenon affects the eye, including retinal blood vessels, is unknown.

Many systemic connective tissue diseases coexist with secondary Sjögren's syndrome - dryness syndrome, which has a well-known ophthalmic aspect [4]. According to the literature, SSc is accompanied by Sjögren's syndrome even in $23.2 \%$ [5], while another study [6] shows the coexistence of these conditions only in $14 \%$. Reviewing latest scientific reports, there can be found more and more papers discussing both ocular symptoms in patients with SSc, their incidence and correlation with the stage of the underlying disease. Although most of the available analyses contain small groups of patients, this allows us to present the most common ophthalmologic pathologies in patients with SSc.

\section{Most common ophthalmic symptoms in patients with systemic sclerosis \\ Eyelids}

Eyelid skin involvement is best documented in the scientific literature and also one of the most common ophthalmic symptoms in patients with SSc. It manifests itself in the stiffness and tightness of the eyelid skin, restriction of eyelid mobility, ectropion, blepharophimosis, eyelid telangiectasia and ciliary madarosis $[7,8]$. Szucs et al. [7] found eyelid changes in 57\% of patients with SSc in their analysis. In a study performed by Ratilglia et al. [9] thickening and hardening of the eyelid skin was observed in $77 \%$ of subjects. Gomes et al. [10] reported changes in eyelids were more common in the form of dcSSc and in patients whose diagnosis of the underlying disease was made at a younger age - on average 35.9 years vs. 44.6 years in patients without eyelid involvement $[8,10]$.

Eyelid abnormalities in the course of SSc are the result of fibrosis processes-accumulation of collagen, mainly of type I, occurring in the connective tissue in the course of the disease [11]. The stiffness of the eyelid skin leads to a limitation of their mobility, which significantly affects the disorder of tear film distribution over the anterior surface of the eye, having a significant impact on the impairment of sharp, clear vision. In turn, lagophthalmos causes excessive loss of tear film resulting from the increased evaporation process. Both disorders lead to the development of chronic dry eye disease (DED) in patients with SSc $[7,10]$.

\section{Conjunctiva}

The conjunctiva due to its rich vascularization is particularly affected by vasculopathy in the course of SSc.
Ophthalmologic examination reveals conjunctival vascular congestion, neovascularization, telangiectasia and varicose vein dilatation [6]. In the analysis of Ratilglia et al. [9], conjunctival tortuosity and dilatation were found in $63 \%$ of 30 examined patients.

On the other hand, in the study by West and Barnett [8], which included 38 patients with SSc, 27 of them (71\%) had vascular sludging within the conjunctiva and 19 (50\%) had dilated vessels. It was also noted that the changes in the conjunctival blood vessels resemble those seen in nailfold capillaroscopy in the course of SSc [8].

In 1993, Mencel et al. [12] conducted a study of conjunctival biopsies of 21 patients with SSc. Microscopic examination of the samples revealed thinning of the conjunctival epithelium and its keratinization, irregularity of epithelial cells and significant loss of goblet cells. The severity of the changes, however, did not show a correlation with the duration of the disease or symptoms of patients in the field of DED. In all biopsies, mast cell infiltration was also found, half of which was in the degranulation phase, indicating the active release of mediators from their granules. Similar changes were also observed in skin and lip biopsies [12]. Based on the studies, it is believed that mast cells may participate in tissue fibrosis in scleroderma [13]. All tested samples showed features of fibrosis beneath the epithelium and around capillaries. Numerous active fibroblasts, collagen fibers grouped in bundles and thickening of capillary walls were observed. Fibrosis was present in conjunctival biopsy in all patients regardless of the duration of the disease and signs, which may serve as a future diagnostic tool in the early stages of the disease [12].

\section{Dry eye disease}

According to many authors, dry eye disease (DED) is the most common ocular manifestation of SSc. In analysis of Szucs et al. [7], DED affected $64.7 \%$ of the 51 patients examined. In turn, in the study of Gomes et al. [10] involving 45 patients, DED was found in $48.9 \%$ of them. DED in the course of SSc is the result of eyelids disorders (which have been described earlier) as well as impairment of the production of tear film itself.

Pathomechanism involves fibrosis in the tear ducts, which leads to a reduction in the production of aqueous layer of tears. In turn, reducing the number of goblet cells of the conjunctival epithelium causing a decrease in the secretion of the tear film mucin layer. It has been studied that in patients with SSc, the velocity of tear secretion is reduced by as much as $67 \%$ compared to the control group [14].

As a result of the aforementioned changes, there is excessive evaporation of tears from the eye surface and their 
quantitative and qualitative deficiency [15]. It has also been proven that the severity of DED in an ophthalmological examination does not correlate with the duration of the underlying disease and the duration of ophthalmic symptoms [12]. Interestingly, in the study of Rentka et al. [16], she showed a weak correlation between signs and symptoms in dry eye syndrome in the course of SSc.

It should not be forgotten that in some patients with SSc, secondary Sjögren's syndrome may coexist giving symptoms of DED. In the previously mentioned study by Mancel et al. [12], biopsies of patients with primary Sjögren's syndrome were also analyzed, showing different background for conjunctival changes in both diseases. In Sjögren's syndrome lymphocytic infiltrates were present in all samples, whereas fibrosis was not observed. It can be presumed that both types of lesions will be present in patients with SSC and secondary Sjögren's syndrome, but this requires further, detailed histopathological examination.

\section{Cornea}

The thickest layer of the cornea is its stroma constituting $90 \%$ of the total thickness. It is made of collagen fibers, mainly collagen type I and $V[17,18]$. In systemic sclerosis, excessive collagen deposition occurs, especially of type I, III and V in the skin and internal organs [19], which led to the hypothesis that corneal thickness in patients with SSc should be increased.

The literature presents several studies verifying this assumption, however, their results are contradictory. Serup et al. [20] in an analysis of 32 patients with SSc showed an increase in corneal thickness, especially in the first 8 years of the disease. However, Gomes et al. [21] did not confirm this, stating no significant difference in corneal thickness in 37 patients with SSc, compared to the control group. Sahin Atik et al. [22] observed a statistically insignificant tendency to decreased corneal thickness in 34 patients with SSc. One of the latest studies, published by Nagy et al. [23], which includes many cofounding factors, supports corneal thickness reduction in patients with SSc compared to the control group.

To conclude, the impact of SSc on the thickness of the cornea requires further, extended studies on larger patient groups. They should include physiological diurnal changes in corneal thickness as well as other potential confounding factors such as gender, race, age and body mass index. It seems reasonable to conduct long-term observation as pathologic changes in the course of SSC appear earlier in tissues with more vascular components.

\section{Pupil diameter}

Studies indicate that the nervous system is also involved in systemic sclerosis. This applies to both central and peripheral nervous system. Studying the pathogenesis of Raynaud's phenomenon, showed increased activity of the sympathetic nervous system while a decrease in the parasympathetic nervous system [24, 25]. Therefore, it was decided to check whether the pupil diameter under the influence of both the parasympathetic and sympathetic nervous system will be different in patients with SSc compared to the control group.

In 2002 and 2003, Bertinotti and del Roso [26, 27] conducted a study of the basal pupil diameter and under the influence of substance $P$ (constricts the pupil by directly affecting the sphincter pupillae muscle). The results showed that the basal diameter of the pupil in patients with SSc was smaller than in the control group with more intense narrowing found to occur under the influence of substance P. In addition, these changes were more evident in patients with limited scleroderma than generalized. The results indicate dysfunctions of the peripheral nervous system especially in IcSSc.

\section{Iris}

The most common iris abnormalities in ophthalmologic examination in patients with SSc are transillumination defect and vascular deformation, which is particularly evident in people with light iris [7, 8]. Szucs et al. [7] observed changes in the iris in $13.7 \%$ of patients while Gomes et al. in 8.9\% [6]. It is believed that the reason for these changes is vasculopathy involving the iris as part of the uvea, which in turn leads to vascular disorders and iris epithelial atrophy [7].

\section{Lens}

The studies conducted so far do not allow to clearly determine the effect of systemic sclerosis on the development of cataract. It seems that glucocorticoid drugs used in some SSc patients may have an effect on the acceleration of lens opacification processes than the underlying disease alone [7, 10]. However, it should be emphasized that modern principles of treatment of patients with SSc practically exclude the use of glucocorticosteroids. These drugs are thought to increase the risk of renal crisis. The use of glucocorticoids in the early stages of the disease (puffy fingers) or in severe arthritis is exceptionally allowed [28].

\section{Retina}

The retina is characterized by immunologically favored microcirculation, lack of resident fibroblasts and adrenergic vasomotor innervation [29, 30]. This structure seems to protect it against vasculopathy and fibrosis processes that occur in systemic sclerosis.

There are many contradictory conclusions in the literature about the effect of SSc on retinal pathologies. 
In some studies, no relationship was found between SSc and retinopathy [31, 32]. Ushiyama et al. [33] analyzed the fundus photos of 29 SSc patients and compared the incidence of retinal abnormalities with the control group and with the result of nailfold capillaroscopy. Retinal changes were found in 34\% of patients with SSc, and in the control group only in $8 \%$ of patients. There was no correlation between abnormal capillaroscopy and retinal lesions in SSc patients. Patients with retinal abnormalities were more likely to have concomitant hypertension. In fundus examination, hard exudates and tortuosity of vessels were most commonly found. Coexisting diabetes and retinopathy, which is the most common cause of retinal hard exudates, were not included in this study.

Cheung et al. [34] found that retinal vascular tortuosity correlates with hypertension and other parameters depending on the type of vessel-venous or arterial, which was not specified in the study by Ushiyama et al. [33]. Therefore, the assessment of the impact of SSc on retinal pathologies requires further study with a larger group of patients, while taking into account cofounding factors, especially hypertension.

Isolated cases of ophthalmic vascular events have also been reported. Konuk et al. [35] presented a case of ocular ischemic syndrome in a 43-year-old patient with dcSSc who had complete stenosis of the internal carotid artery and, as a consequence, ipsilateral loss of vision. In turn, Raja et al. [36] described a case of a 75-year-old patient with IcSSc who developed central retinal artery embolism, leading to unilateral loss of vision.

\section{Choroid}

The choroid is the most vascularized part of the eye, and for this reason it was thought that vasculopathy in the course of SSc would also affect it to a significant extent. Histopathological studies conducted by MacLean and Guthrie [37] showed thickening of the precapillary base-

Table I. Incidence of ocular findings in patients with systemic sclerosis

\begin{tabular}{|lc|}
\hline Ocular findings & Percent (\%) \\
\hline Eyelid (stiffness, tightness, telangiectasia) & $51.1-77.0$ \\
\hline $\begin{array}{l}\text { Conjunctiva (neovascularization, } \\
\text { teleangiectasia, varicose vein dilatation) }\end{array}$ & $15.7-63.0$ \\
\hline Dry eye disease & $48.9-64.7$ \\
\hline Iris (transilumination, vascular deformation) & $8.9-13.7$ \\
\hline Cataract & $42.2-51.0$ \\
\hline Retina (hard exudates, tortuosity of vessels) & $10.0-34.0$ \\
\hline Choroid (perfusion abnormalities) & $33.0-50.0$ \\
\hline Glaucoma & $11.1-21.6$ \\
\hline
\end{tabular}

ment membrane, swelling of endothelial cells and obliteration of small vessel lumen and areas of choroidal ischemia. The research of Grennan et al. [32] using fluorescein angiography confirmed choroidal perfusion disorders. In this study, areas of choroidal non-perfusion were observed in up to $50 \%$ of subjects. This affected all patients with the aggressive form of dcSSc, but also a few patients with a milder form, where the skin lesions concerned only fingers. Coşkun et al. [38] in their study using optical coherence tomography (OCT) found significant choroid thinning in SSc patients compared to the control group.

In 2019, Ranjbar et al. [39] using angio-OCT described in patients with systemic sclerosis reduced perfusion in all layers of the choroid in the submacular region. These studies included confounding factors such as patient age, body mass index, and axial length of the eyeball, which also affect choroidal thickness.

\section{Glaucoma}

The relationship between SSC and the incidence of glaucoma has been analyzed in several studies. Gomes et al. [10] found the presence of open angle glaucoma in $11.1 \%$ of subjects, which gives a higher frequency than in the general population (2.4\% for the Brazilian population). The authors suggest that the increased incidence of glaucoma in SSc patients may be due to vasculopathy, which in this case would involve perfusion and circulation in the blood vessels of the optic nerve. Allanore et al. [40] analyzed 61 patients with SSc and found between them significantly more frequent glaucoma changes than in the control group. The described deviations included a cup/disc area of the optic disc ratio $>0.3$ and changes in the field of view. Intraocular pressure in all patients was within the accepted norm and therefore a relationship between SSc and normal pressure glaucoma was concluded.

However, Chan and Liu [41] believe that the Allanore study contains methodological errors in the analysis of field of view results, and also includes only one measurement of intraocular pressure. Therefore, the increased risk of glaucoma in SSc patients requires further research.

Incidence of ocular findings in patients with SSc is presented in Table I.

\section{Pathogenesis of ocular involvement in patients with systemic sclerosis}

The pathogenesis of ocular involvement, as well as the pathogenesis of changes in internal organs and the involvement of body integumentary system in SSC patients is complex and poorly understood. The cooperation of three groups of mechanisms is assumed [42]. 
These include vascular changes, immune disorders and fibrosis. It is often assumed that the fibrosis process is an advanced stage of the disease and the activation of fibroblasts combined with excessive production of extracellular components of connective tissue is a common descent of vascular changes and altered immune-inflammatory processes.

In SSc patients early in the disease, including the preclinical stages, numerous autoantibodies are detected. They are quite closely related to the clinical profile and course of the disease. Patients have stable (constitutional) activation and an increased number of B-CD19 cells accompanied by a decrease in the number of B-CD22 cells. In addition to autoantibodies directed against cellular components, there are also antibodies directed to adhesion proteins, endothelin $A$ receptor, angiotensin II receptors and endothelial cells. Autoantibodies are thought to be involved in the development of vascular lesions, including leading to damage to endothelial cells. It is associated with the activation of vascular wall cells and the proliferation of myofibroblasts of the middle layer of small vessels. Activation of the fibrosis process can occur in different ways.

The role of hypoxia and oxygen free radicals, release of growth factors (primarily profibrotic growth factors: transforming growth factor $\beta$ - TGF- $\beta$, connective tissue growth factor - CTGF, platelet-derived growth factor PDGF) and pro-inflammatory factors (interleukin 6) and impaired apoptosis of cells regulating the deposition of extracellular substance components [43]. However, it should be noted that SSc, unlike other systemic connective tissue diseases, is characterized by relatively low inflammation. An increase in inflammatory markers is found only in the early stages of the disease and with late infectious complications [44].

Ocular manifestation of the disease can reflect all of these processes, including vascular, autoimmune, inflammatory ones and excessive deposition of connective tissue components. Attempts are made to distinguish primary changes associated with the pathogenesis of the disease and secondary changes. Secondary changes may result from complications (e.g. bacterial or viral infections), and may also be associated with the treatment being used.

Contemporary SSc therapy is based on the use of treatment targeted at individual organs. Immunosuppressive and antiproliferative drugs are used. Immunoablation with autologous stem cell transplantation is considered to be the most effective therapy, but is unfortunately only applicable to some patients [45]. The treatment procedures can have a direct or indirect effect on the organ of vision. Of course, SSc patients may have ophthalmic disease as incidental comorbidities.

\section{Conclusions}

In order to draw reliable conclusions about the impact of SSc on the eye, further standardized studies with more numerous groups of patients are required. It would also be necessary to prove clear correlations between ophthalmic changes, their type and severity, and the progression of systemic sclerosis. The dependence of the autoantibody profile, the severity of skin fibrosis, the development of organ changes as well as the severity of microcirculation changes and the severity of the inflammatory process on the occurrence and severity of changes in the organ of vision are unknown.

The impact of the treatment may also be significant. Another issue is the hypothetical possibility of early detection of SSc or the determination of disease activity or its progression and prognosis based on the analysis of ocular changes.

To summarize the above analysis, currently the best documented ophthalmic manifestations in SSc are eyelid, conjunctival, dry eye syndrome and choroidal perfusion disorders. They result from fibrosis and vasculopathy, which are the main features of the underlying disease. Ocular involvement occurs in many rheumatological diseases, hence clinicians should be vigilant not to miss the ophthalmological pathology in the course of SSc and ensure adequate treatment is provided at the appropriate time.

The authors declare no conflict of interest.

\section{References}

1. Varga J, Denton CP, Wigley FM, et al. Scleroderma: from pathogenesis to comprehensive management, $2^{\text {nd }}$ ed. Springer, New York 2017.

2. Hachulla E, Czirják L (eds.). EULAR Textbook on systemic sclerosis. BMJ Publishing Group, London 2013.

3. Kucharz EJ, Kopeć-Mędrek M. Systemic sclerosis sine scleroderma. Adv Clin Exp Med 2017; 26: 875-880, DOI: 10.17219/ acem/64334.

4. Bellando-Randone S, Matucci-Cerinic M. Very Early Systemic Sclerosis and Pre-systemic Sclerosis: definition, recognition, clinical relevance and future directions. Curr Rheumatol Rep 2017; 19: 65, DOI: 10.1007/s11926-017-0684-2.

5. Horimoto AMC, de Macedo Possamai V, da Costa IP. Sjögren's Syndrome and Sicca Symptoms in Patients with Systemic Sclerosis. J Arthritis 2016; 5: 190, DOI: 10.4172/2167-7921.1000190.

6. Avouac J, Sordet C, Depinay C, et al. Systemic sclerosis-associated Sjögren's syndrome and relationship to the limited cutaneous subtype: Results of a prospective study of sicca syndrome in 133 consecutive patients. Arthritis Rheum 2006; 54: 2243-2249, DOI: 10.1002/art.21922.

7. Szucs G, Szekanecz Z, Aszalos Z, et al. A wide spectrum of ocular manifestations signify patients with systemic sclerosis. Ocul Immunol Inflamm 2019, DOI: 10.1080/09273948.2019.1657467. 
8. West RH, Barnett AJ. Ocular involvement in scleroderma. Br J Ophthalmol 1979; 63: 845-847, DOI: 10.1136/bjo.63.12.845.

9. Ratiglia R, Scorza R, Santori M, et al. Altérations oculaires au cours de la sclérodermie systèmique. Ophtalmologie 1987; 1: 527-529.

10. Gomes Bde A, Santhiago MR, Magalhães P, et al. Ocular findings in patients with systemic sclerosis. Clinics (Sao Paulo) 2011; 66: 379-385, DOI: 10.1590/s1807-59322011000300003.

11. Jinnin M. Mechanisms of skin fibrosis in systemic sclerosis. J Dermatol 2010; 37: 11-25, DOI: 10.1111/j.1346-8138.2009. 00738.x.

12. Mancel E, Janin A, Gosset D, et al. Conjunctival biopsy in scleroderma and primary Sjögren's syndrome. Am J Ophthalmol 1993; 115: 792-799, DOI: 10.1016/s0002-9394(14)73650-5.

13. Hügle $T$, Hogan V, White KE, van Laar JM. Mast cells are a source of transforming growth factor $\beta$ in systemic sclerosis. Arthritis Rheum 2011; 63: 795-799. DOI: 10.1002/art.30190.

14. Rentka A, Hársfalvi J, Berta A, et al. Vascular Endothelial Growth Factor in Tear Samples of Patients with Systemic Sclerosis. Mediators Inflamm 2015; 2015: 573681, DOI: 10.1155/2015/573681.

15. Waszczykowska A, Goś R, Waszczykowska E, et al. Prevalence of ocular manifestations in systemic sclerosis patients. Arch Med Sci 2013; 9: 1107-1113, DOI: 10.5114/aoms.2013.39217.

16. Rentka A, Nagy A, Harsfalvi J, et al. Association between objective signs and subjective symptoms of dry eye disease in patients with systemic sclerosis. Rheumatol Int 2017; 37 : 1835-1845, DOI: 10.1007/s00296-017-3794-2.

17. Kucharz EJ. The collagens: biochemistry and pathophysiology Springer Verlag, Berlin-Heidelberg-New York 1992.

18. Ihanamäki T, Pelliniemi LJ, Vuorio E. Collagens and collagenrelated matrix components in the human and mouse eye. Prog Retin Eye Res 2004; 23: 403-434, DOI: 10.1016/j.preteyeres.2004.04.002.

19. Ho Y, Lagares D, Tager A, et al. Fibrosis - a lethal component of systemic sclerosis. Nat Rev Rheumatol 2014; 10: 390-402, DOI: 10.1038/nrrheum.2014.53.

20. Serup L, Serup J, Hagdrup HK. Increased central cornea thickness in systemic sclerosis. Acta Ophthalmol (Copenh) 1984 62: 69-74, DOI: 10.1111/j.1755-3768.1984.tb06758.x.

21. Gomes Bde A, Santhiago MR, Kara-Junior N, et al. Central corneal thickness in patients with systemic sclerosis: a controlled study. Cornea 2011; 30: 1125-1128, DOI: 10.1097/ICO.0b013e318206cac1.

22. Sahin Atik S, Koc F, Akin Sari S, et al. Anterior segment parameters and eyelids in systemic sclerosis. Int Ophthalmol 2016; 36: 577-583, DOI: 10.1007/s10792-015-0165-4.

23. Nagy A, Rentka A, Nemeth G, et al. Corneal manifestations of systemic sclerosis. Ocul Immunol Inflamm 2019; 27: 968-977, DOI: 10.1080/09273948.2018.1489556.

24. Cerinic MM, Generini S, Pignone A, Casale R. The nervous system in systemic sclerosis (scleroderma). Clinical features and pathogenetic mechanisms. Rheum Dis Clin North Am 1996; 22: 879-892, DOI: 10.1016/s0889-857x(05)70306-9.

25. Kahaleh B, Matucci-Cerinic M. Raynaud's phenomenon and scleroderma. Dysregulated neuroendothelial control of vascular tone. Arthritis Rheum 1995; 38: 1-4, DOI: 10.1002/art. 1780380102.

26. Del Rosso A, Bertinotti L, Pietrini U, et al. Pupillocynetic activity of substance P in systemic sclerosis. J Rheumatol 2003; 30: 1231-1237.
27. Bertinotti L, Pietrini U, Del Rosso A, et al. The use of pupillometry in joint and connective tissue diseases. Ann N Y Acad Sci 2002; 966: 446-455, DOI: 10.1111/j.1749-6632.2002.tb04246.x.

28. Kucharz EJ. Twardzina układowa. In: Stany nagłe w reumatologii, Kotyla P (ed.). Via Medica, Gdańsk 2020 [In print].

29. Aissopou EK, Bournia VK, Protogerou AD, et al. Intact calibers of retinal vessels in patients with systemic sclerosis. J Rheumatol 2015; 42: 608-613, DOI: 10.3899/jrheum.141425.

30. Streilein J. Ocular immune privilege: therapeutic opportunities from an experiment of nature. Nat Rev Immunol 2003; 3: 879889, DOI: $10.1038 /$ nri1224.

31. Kirkham TH. Scleroderma and Sjögren's syndrome. Br J Ophthalmol 1969; 53: 131-133, DOI: 10.1136/bjo.53.2.131.

32. Grennan DM, Forrester J. Involvement of the eye in SLE and scleroderma. A study using fluorescein angiography in addition to clinical ophthalmic assessment. Ann Rheum Dis 1977; 36: 152-156, DOI: 10.1136/ard.36.2.152.

33. Ushiyama O, Ushiyama K, Yamada T, et al. Retinal findings in systemic sclerosis: a comparison with nailfold capillaroscopic patterns. Ann Rheum Dis 2003; 62: 204-207, DOI: 10.1136/ ard.62.3.204.

34. Cheung CY, Zheng Y, Hsu W, et al. Retinal vascular tortuosity, blood pressure, and cardiovascular risk factors. Ophthalmology 2011; 118: 812-818, DOI: 10.1016/j.ophtha.2010.08.045

35. Konuk O, Ozdek S, Onal B, et al. Ocular ischemic syndrome presenting as central retinal artery occlusion in scleroderma. Retina 2006; 26: 102-104, DOI: 10.1097/00006982-200601000-00017.

36. Raja MS, Marshall T, Burton BJ. Acute central retinal artery occlusion presenting as CREST syndrome: a case report. Cases I 2009; 2: 9, DOI: 10.1186/1757-1626-2-9.

37. 37. Maclean H, Guthrie W. Retinopathy in scleroderma. Trans Ophthalmol Soc U K 1970; 89: 209-220.

38. Coşkun E, Zengin O, Kenan S, et al. Evaluation of choroidal thickness in patients with scleroderma. Eye (Lond) 2016; 30: 588-592, DOI: 10.1038/eye.2015.287.

39. Ranjbar M, Rothe M, Klapa S, et al. Evaluation of choroidal substructure perfusion in patients affected by systemic sclerosis: an optical coherence tomography angiography study. Scand J Rheumatol 2020; 49: 141-145, DOI: 10.1080/03009742.2019. 1641616.

40. Allanore Y, Parc C, Monnet D, et al. Increased prevalence of ocular glaucomatous abnormalities in systemic sclerosis. Ann Rheum Dis 2004; 63: 1276-1278, DOI: 10.1136/ard.2003.013540.

41. Chan AY, Liu DT. Increased prevalence of ocular glaucomatous abnormalities in systemic sclerosis. Ann Rheum Dis 2005; 64: 341-342.

42. Furue M, Mitoma C, Mitoma $\mathrm{H}$, et al. Pathogenesis of systemic sclerosis-current concept and emerging treatments. Immunol Res 2017; 65: 790-797, DOI: 10.1007/s12026-017-8926-y.

43. Kuźnik-Trocha K, Winsz-Szczotka K, Komosińska-Vassev K, et al. Plasma glycosaminoglycan profiles in systemic sclerosis: associations with MMP-3, MMP-10, TIMP-1, TIMP-2, and TGFbeta. Biomed Res Int 2020; 2020: 6416514, DOI: 10.1155/2020/ 6416514.

44. Kucharz E, Grucka-Mamczar E, Mamczar A, et al. Acute-phase proteins in patients with systemic sclerosis. Clin Rheumatol 2000; 19, 165-166, DOI: 10.1007/s100670050039.

45. Denton CP, Khanna D. Systemic sclerosis. Lancet 2017; 390: 1685-1699, DOI: 10.1016/S0140-6736(17)30933-9. 